\title{
Sequential expression of neutrophil chemoattractants in cerebrospinal fluid after subarachnoid hemorrhage
}

Koji Osuka ( $\square$ kosuka@aichi-med-u.ac.jp )

Aichi Medical University School of Medicine https://orcid.org/0000-0002-6301-3189

Yasuo Watanabe

Showa Pharmaceutical University: Showa Yakka Daigaku

Chiharu Suzuki

Aichi Medical University: Aichi Ika Daigaku

Kenichiro Iwami

Aichi Medical University: Aichi Ika Daigaku

Shigeru Miyachi

Aichi Medical University: Aichi Ika Daigaku

\section{Short report}

Keywords: Neutrophil chemoattractants, neutrophil gelatinase-associated lipocalin, Myeloperoxidase, Subarachnoid hemorrhage

Posted Date: February 22nd, 2021

DOl: https://doi.org/10.21203/rs.3.rs-205543/v1

License: (c) (1) This work is licensed under a Creative Commons Attribution 4.0 International License. Read Full License 


\section{Abstract}

Background:

Neutrophils induce inflammation through the exocytosis of cytotoxic granule proteins. Recently, neutrophils have been reported to be an independent parameter associated with unfavorable outcomes after subarachnoid hemorrhage (SAH). However, the mechanism by which neutrophils accumulate within the CSF after SAH remains undetermined.

\section{Findings:}

Concentrations of C5a, epithelial neutrophil activating peptide 78 (ENA-78), interleukin-8 (IL-8), growthregulated oncogene- $a($ GRO- $a$ ), neutrophil gelatinase-associated lipocalin (NGAL) and myeloperoxidase (MPO) were measured serially until day 14 in the CSF of 10 patients with SAH. CSF samples obtained from patients suffering from unruptured aneurysms were used as controls. The concentrations of $\mathrm{C} 5 \mathrm{a}$ and ENA-78 were significantly increased on day 1, while those of IL-8 and GRO-a significantly increased during days 3-7 compared with those of the control samples. The levels of NGAL and MPO, components of neutrophil granules, significantly increased during days 1 5 after SAH and gradually decreased thereafter. The correlations between ENA-78 and C5a on day 1, IL-8 and GRO- $a$ on days 3 7, and NGAL and MPO on days 1 3 were significant.

Conclusions:

These neutrophil chemoattractants might be serially involved in the infiltration of neutrophils into the CSF after SAH. Migrated neutrophils play an important role in inflammatory reactions in the central nervous system after SAH.

\section{Introduction}

There is growing evidence supporting the role of inflammation in central nervous system diseases. Leukocyte recruitment to the site of inflammation is the initial event, and infiltrating neutrophils play an important role in inflammation. Polymorphonuclear leukocytes (PMNLs) intensively accumulate in regions of cerebral ischemia, which is correlated with the severity of brain tissue damage and poor neurological outcomes [1]. Higher neutrophils, lower lymphocytes and a higher neutrophil-to-lymphocyte ratio (NLR) were independently associated with poor intracerebral hemorrhage outcome [2]. In the case of subarachnoid hemorrhage, the NLR also represents an independent predictor of unfavorable functional outcomes [3]. Furthermore, neutrophils on day 3 in CSF were significantly associated with cerebral vasospasm [4]. However, how these neutrophils migrate into CSF after SAH remains unknown. The present study was therefore undertaken to clarify the time course of the expression of neutrophilchemoattractant chemokines in the CSF after SAH.

\section{Methods}




\section{Patients and control subjects}

Ten patients who underwent surgical obliteration of cerebral aneurysms at Aichi Medical University Hospital within 1 day after the onset of SAH were enrolled in this study. The patients ranged in age from 41 to 66 years (mean 53 years); 6 were men, and 4 were women. Initial clinical status was classified according to the Hunt and Kosnik grade; 4 patients were grade II, and 6 were grade III. The amount of blood in the subarachnoid space was defined by Fisher's criteria; 7 patients were group 3 , and 3 were group 4. Locations of all aneurysms were in the anterior circulation. After aneurysms were clipped, a cisternal catheter was inserted into the chiasmatic or prepontine cistern, and postoperative CSF samples were collected serially with a drainage tube over the following 14 days. One patient developed delayed ischemic neurological deficits. In all the other patients, the postoperative course was uneventful. No patients developed infection. As a control, CSF samples were obtained from 8 patients (mean 62 years) undergoing neck clipping for unruptured cerebral aneurysms. All samples were immediately centrifuged, and supernatant fluids were stored at $-80^{\circ} \mathrm{C}$ until analysis. The Ethics Committee of Aichi Medical University approved this clinical experiment.

\section{Analysis of CSF}

C5a (C5a + C5a des-arg) (Quidel Corporation, San Diego, CA, USA), epithelial neutrophil activating peptide 78 (ENA-78, Thermo Fisher Scientific, Inc., Waltham, MA USA), interleukin-8 (IL-8, Thermo Fisher Scientific, Inc.), growth-regulated oncogene-a (GRO-a, Thermo Fisher Scientific, Inc.), neutrophil gelatinaseassociated lipocalin (NGAL, R\&D Systems, Inc., Minneapolis, MN, USA) and myeloperoxidase (MPO, R\&D Systems, Inc.) were measured using enzyme-linked immunosorbent assay (ELISA) kits according to the manufacturer's instructions. The limits of the minimum detectable dose of these respective assays were $0.01 \mathrm{ng} / \mathrm{ml}$ for C5a, $10 \mathrm{pg} / \mathrm{ml}$ for ENA-78, $1.3 \mathrm{pg} / \mathrm{ml}$ for IL-8, $7.6 \mathrm{pg} / \mathrm{ml}$ for GRO-a, $0.012 \mathrm{ng} / \mathrm{ml}$ for NGAL and $0.014 \mathrm{ng} / \mathrm{ml}$ for MPO.

\section{Statistical analysis}

Statistical differences between the groups were assessed by one-way ANOVA followed by Fisher's post hoc test. Correlations between cytokine and chemokine levels were assessed by Pearson's correlation coefficient. Statistical significance was defined at the $p<0.05$ level.

\section{Results}

\section{Change in C5a, ENA-78, IL-8, GRO-a, NGAL and MPO after SAH}

The concentrations of C5a and ENA-78 were significantly increased on day 1 and gradually decreased thereafter (Fig. 1a and b, respectively). The concentrations of IL-8 and GRO-a significantly increased during days 3-7 compared with those of the control samples (Fig. 1c and d, respectively). The NGAL and MPO levels significantly increased during days 1 5 after SAH and decreased thereafter (Fig. 1e and f, respectively). 


\section{Correlations between C5a, chemokine, NGAL and MPO}

The concentrations of ENA-78 and C5a on day 1 were positively correlated with each other according to Pearson's correlation coefficient (Fig. 2a). The levels of GRO-a and IL-8 were also positively correlated with each other on days $3 \sim 7$ according to Pearson's correlation coefficient (Fig. 2b). Moreover, those of NGAL and MPO were positively correlated with each other on days $1 \sim 3$ according to Pearson's correlation coefficient (Fig. 2c).

\section{Discussion}

In this study, we showed that the concentrations of C5a and ENA-78 in CSF significantly increased immediately after the onset of SAH. Thereafter, IL-8 and GRO-a significantly increased on days $3 \sim 7$. NGAL and MPO significantly increased after the onset of SAH until day 5 . The correlations between ENA78 and C5a on day 1, IL-8 and GRO-a on days $3 \sim 7$, and NGAL and MPO on days $1 \sim 3$ were statistically significant.

Complement $\mathrm{C5a}$ is rapidly cleaved into the more stable, less active C5a des-arg. C5a ELISA kit from Quidel Corporation can detect both $\mathrm{C5}$ a and C5a des-arg. C5a and C5a des-arg have been demonstrated to act primarily on neutrophils to stimulate adherence to the endothelium in vitro [5]. Previously, concentrations of C3a and C4a in the CSF were shown to be significantly elevated on days $0 \sim 1$ after SAH and decreased rapidly [6].The complement cascade in CSF becomes activated on days $0 \sim 2$ after SAH following blood-brain barrier damage in Man [7]. These time courses of complement expression are in agreement with our results. After $\mathrm{SAH}$, early $\mathrm{C} 3 \mathrm{a}$ and $\mathrm{C} 5 \mathrm{a}$ levels in plasma were increased compared with those in the control patients, especially the early C3a levels, which are a strong predictor of functional outcome [8]. The concentrations of ENA-78 in CSF were significantly higher than those in the control during the first $24 \mathrm{hr}$ after ischemic stroke and correlated positively with the volume of early brain damage [9]. ENA-78 is elevated in CSF with bacterial meningitis and mediates chemotactic activity on neutrophils in vitro [10]. ENA-78, similar to IL-8, plays an important role in the recruitment of neutrophils into inflamed joints of rheumatoid arthritis patients [11]. Considering our data, C5a, C5a des-arg and ENA78 are the main factors inducing neutrophils into CSF at the first stage after the onset of SAH.

IL-8 induces chemotaxis in neutrophils, causing them to accumulate at sites of inflammation [12]. The expression of IL-8 mRNA in the rabbit basilar artery significantly increased on days $4 \sim 7$ after a single injection model of SAH [13], which is in agreement with our time course of expression of IL-8. GRO-a, a protein structurally related to IL-8, induces chemotaxis, exocytosis and the respiratory burst of neutrophils [14]. To our knowledge, this is the first report of serial changes in GRO-a in CSF after SAH, which correlate well with the expression of IL-8. Neutrophils migrate into tissues after activation, where they can survive only for 1-2 days. These chemokines play an important role in the continuous induction of neutrophils on days $4 \sim 7$ and may be involved in the vasospasm and detrimental effects of SAH.

MPO and NGAL are present in azurophils and specific granules in neutrophil polymorphonuclear leukocytes, respectively [15]. Urinary NGAL, reflecting tubular cell injury, is considered a sensitive marker 
for acute kidney injury [16]. NGAL sensitizes nerve cells to $\beta$-amyloid toxicity and acts as a molecular actor in neuroinflammation in the early clinical stages of Alzheimer's disease [17]. MPO contributes to atherogenesis by generating the cytotoxic oxidants hypochlorous acid $(\mathrm{HOCl})$ and tyrosyl radicals in the vascular wall [18]. Our finding that a significant correlation exists between the concentration of NGAL and MPO in CSF on days $1 \sim 3$ suggested that previous neutrophilic chemokines induced the migration of neutrophils into the CSF and played an important role in neuroinflammation in the central nervous system.

Our study is a preliminary analysis of ten patients. More data are necessary to determine the precise role of these chemokines and the correlations between the levels of chemokines and patient outcomes. Exploring the origin and expression site of these chemokines after SAH is also needed to establish potential therapeutic strategies targeting neutrophils.

\section{Conclusions}

The present investigation, for the first time, clarified the chronological changes in the concentrations of neutrophil-chemoattractant chemokines in the CSF after SAH. Of particular note is our finding that $\mathrm{C} 5 \mathrm{a}$ and ENA-78 were significantly expressed on day 1 in the CSF after SAH; thereafter, IL-8 and GRO-a appeared on days $3 \sim 7$ in the CSF after SAH. There was a correlation between these factors. NGAL and MPO significantly increased on days $1 \sim 3$, suggesting the footprint of neutrophils in the CSF. Further studies using an antagonist of receptors of neutrophilic chemokines should be conducted to more precisely clarify the roles of neutrophils after SAH.

\section{Abbreviations}

ENA-78: epithelial neutrophil activating peptide 78; GRO-a: growth-regulated oncogene-a; IL-8: interleukin8; MPO; myeloperoxidase; NGAL: neutrophil gelatinase-associated lipocalin

\section{Declarations}

\section{Acknowledgments}

None.

\section{Authors' contributions}

KO and YW: study concept and design, statistical analysis, manuscript preparation. CS and Kl: data acquisition and processing. SM: project execution and manuscript critique. All authors read and approved the final manuscript and agree with its submission for publication.

\section{Funding}

This work was supported by JSPS KAKENHI Grant Numbers JP17K10853 (K. Osuka). 
Availability of data and material

The dataset supporting the conclusions of this article is included with in the article.

\section{Ethics approval and consent to participate}

The study was conducted with approval of the Aichi Medical University Ethics Committee. Written informed consent was obtained from the patient for this study.

\section{Consent for publication}

Not applicable.

\section{Competing interests}

The authors declare that they have no competing interests.

\section{References}

1. Akopov SE, Simonian NA, Grigorian GS. Dynamics of polymorphonuclear leukocyte accumulation in acute cerebral infarction and their correlation with brain tissue damage. Stroke. 1996;27:1739-43.

2. Lattanzi S, Cagnetti C, Provinciali L, Silvestrini M. Neutrophil-to-Lymphocyte Ratio Predicts the Outcome of Acute Intracerebral Hemorrhage. Stroke. 2016;47:1654-7.

3. Giede-Jeppe A, Reichl J, Sprügel MI, Lücking H, Hoelter P, Eyüpoglu IY, Kuramatsu JB, Huttner HB, Gerner ST. Neutrophil-to-lymphocyte ratio as an independent predictor for unfavorable functional outcome in aneurysmal subarachnoid hemorrhage. Journal of neurosurgery. 2019;132:400-7.

4. Provencio JJ, Fu X, Siu A, Rasmussen PA, Hazen SL, Ransohoff RM. CSF neutrophils are implicated in the development of vasospasm in subarachnoid hemorrhage. Neurocritical care. 2010;12:244-51.

5. Tonnesen MG, Smedly LA, Henson PM. Neutrophil-endothelial cell interactions. Modulation of neutrophil adhesiveness induced by complement fragments $\mathrm{C} 5 \mathrm{a}$ and $\mathrm{C} 5 \mathrm{a}$ des arg and formylmethionyl-leucyl-phenylalanine in vitro. J Clin Investig. 1984;74:1581-92.

6. Kasuya $\mathrm{H}$, Shimizu T. Activated complement components $\mathrm{C} 3 \mathrm{a}$ and $\mathrm{C} 4 \mathrm{a}$ in cerebrospinal fluid and plasma following subarachnoid hemorrhage. Journal of neurosurgery. 1989;71:741-6.

7. Lindsberg PJ, Ohman J, Lehto T, Karjalainen-Lindsberg ML, Paetau A, Wuorimaa T, Carpén O, Kaste $\mathrm{M}$, Meri S. Complement activation in the central nervous system following blood-brain barrier damage in man. Ann Neurol. 1996;40:587-96.

8. Mack WJ, Ducruet AF, Hickman ZL, Garrett MC, Albert EJ, Kellner CP, Mocco J, Connolly ES. Jr. Early plasma complement C3a levels correlate with functional outcome after aneurysmal subarachnoid hemorrhage. Neurosurgery. 2007;61:255 - 60; discussion $60-1$..

9. Zaremba J, Skrobański P, Losy J. The level of chemokine CXCL5 in the cerebrospinal fluid is increased during the first 24 hours of ischaemic stroke and correlates with the size of early brain 
damage. Folia Morphol. 2006;65:1-5.

10. Zwijnenburg PJ, de Bie HM, Roord JJ, van der Poll T, van Furth AM. Chemotactic activity of CXCL5 in cerebrospinal fluid of children with bacterial meningitis. J Neuroimmunol. 2003;145:148-53.

11. Koch AE, Kunkel SL, Harlow LA, Mazarakis DD, Haines GK, Burdick MD, Pope RM, Walz A, Strieter RM. Epithelial neutrophil activating peptide-78: a novel chemotactic cytokine for neutrophils in arthritis. $J$ Clin Investig. 1994;94:1012-8.

12. Baggiolini M, Clark-Lewis I. Interleukin-8, a chemotactic and inflammatory cytokine. FEBS Lett. 1992;307:97-101.

13. Wang Y, Zhong M, Tan XX, Yang YJ, Chen WJ, Liu W, Zheng K. Expression change of interleukin-8 gene in rabbit basilar artery after subarachnoid hemorrhage. Neurosci Bull. 2007;23:151-5.

14. Geiser T, Dewald B, Ehrengruber MU, Clark-Lewis I, Baggiolini M. The interleukin-8-related chemotactic cytokines GRO alpha, GRO beta, and GRO gamma activate human neutrophil and basophil leukocytes. J Biol Chem. 1993;268:15419-24.

15. Borregaard N, Cowland JB. Granules of the human neutrophilic polymorphonuclear leukocyte. Blood. 1997;89:3503-21.

16. Bolignano D, Donato V, Coppolino G, Campo S, Buemi A, Lacquaniti A, Buemi M. Neutrophil gelatinase-associated lipocalin (NGAL) as a marker of kidney damage. American journal of kidney diseases: the official journal of the National Kidney Foundation. 2008;52:595-605.

17. Naudé PJ, Nyakas C, Eiden LE, Ait-Ali D, van der Heide R, Engelborghs S, Luiten PG, De Deyn PP, den Boer JA, Eisel UL. Lipocalin 2: novel component of proinflammatory signaling in Alzheimer's disease. FASEB journal: official publication of the Federation of American Societies for Experimental Biology. 2012;26:2811-23.

18. Daugherty A, Dunn JL, Rateri DL, Heinecke JW. Myeloperoxidase, a catalyst for lipoprotein oxidation, is expressed in human atherosclerotic lesions. J Clin Investig. 1994;94:437-44.

\section{Figures}



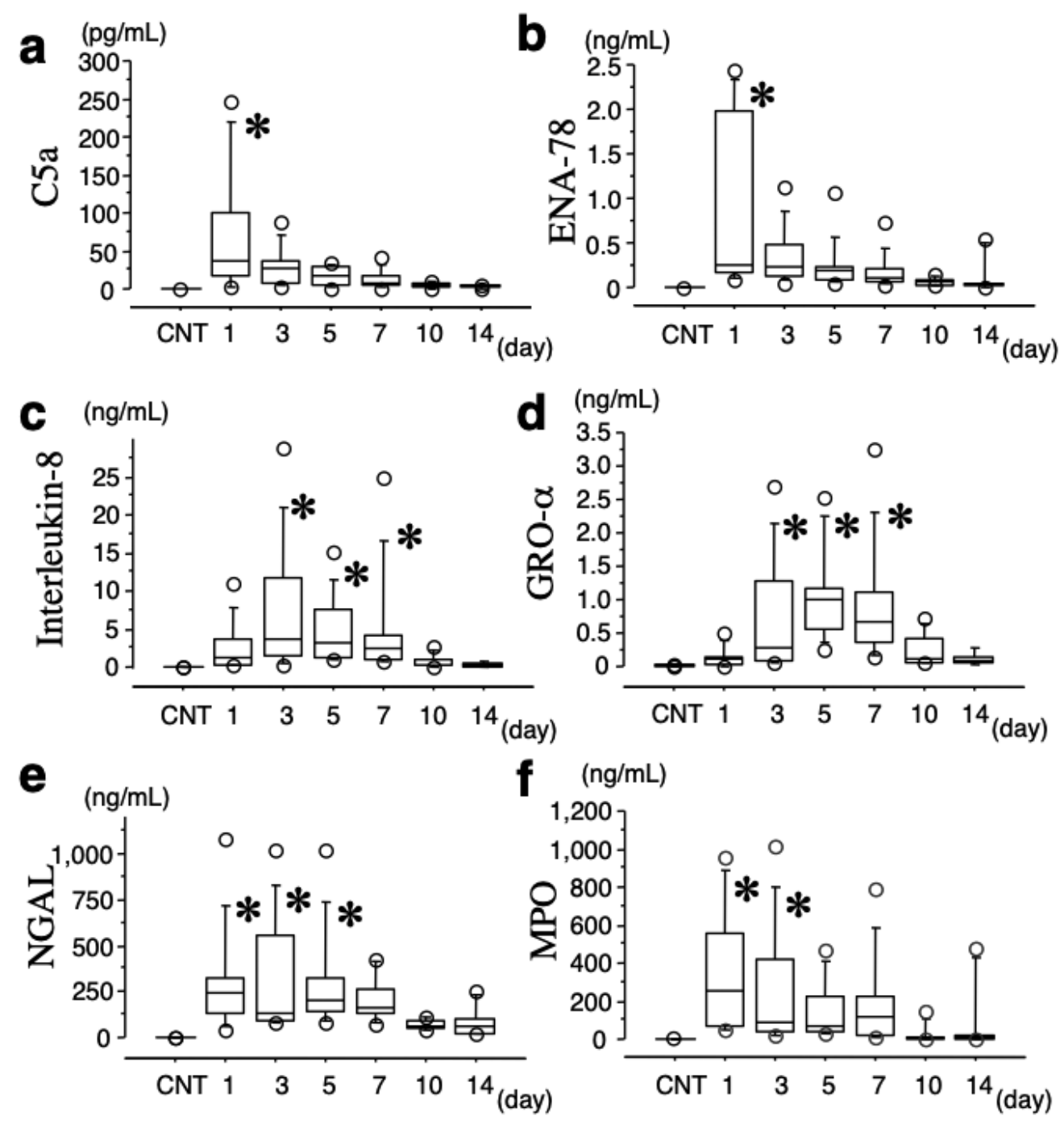

Fig. 1

\section{Figure 1}

Serial changes in complement C5a(a), ENA-78 (b), interleukin-8 (c), GRO-a (d), NGAL (e), and MPO (f) in the CSF from 10 patients with subarachnoid hemorrhage. Data are represented as box-and-whisker plots indicating the median, the upper and lower quartiles, and the highest and the lowest values. CNT: CSF from unruptured intracranial aneurysm. ${ }^{*} \mathrm{p}<0.05$ compared with CNTs by one-way ANOVA followed by Fisher's post hoc test. 

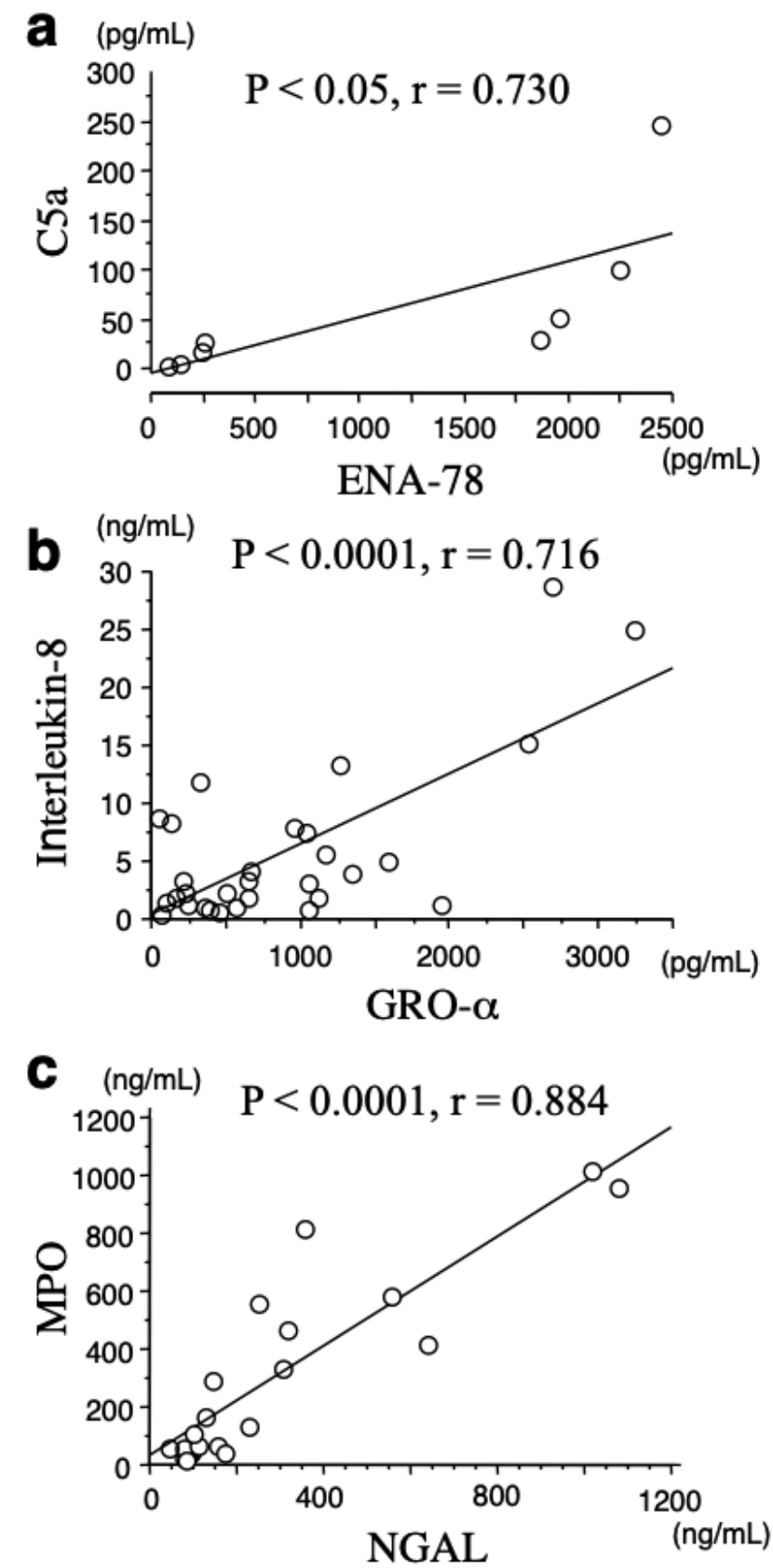

Fig. 2

\section{Figure 2}

The concentrations of ENA-78 and C5a on day 1 (a), GRO- $a$ and interleukin-8 on days $3 \sim 7$ (b) and NGAL and MPO on days $1 \sim 3$ (c) were positively correlated with each other according to Pearson's correlation coefficient. 